\title{
Surgical Landmarks of the Endoscopic Transcanal Transcochlear Approach: A Cadaveric Study
}

\author{
Saleh Mohebbi ${ }^{1}$, Ahmad Daneshi ${ }^{1}$, Alimohamad Asghari ${ }^{2}$, Mohammad Mohseni ${ }^{1}$, Mohammad Arya \\ Sadr ${ }^{1,{ }^{*}}$ and Hessam Eskandarzadeh ${ }^{1}$ \\ ${ }^{1}$ ENT and Head and Neck Research Center and Department, The Five Senses Institute, Iran University of Medical Sciences, Tehran, Iran \\ ${ }^{2}$ Skull Base Research Center, The Five Senses Institute, Iran University of Medical Sciences, Tehran, Iran \\ "Corresponding author: Head and Neck Surgery, ENT and Head and Neck Research Center, School of Medicine, Iran University of Medical Sciences, Tehran, Iran. Email: \\ mohammadpapi1987@gmail.com
}

Received 2019 June 01; Revised 2019 September 17; Accepted 2019 October 09.

\begin{abstract}
Background: The lateral skull base lesions are one of the most challenging parts of the head and neck. Surgical approaches for this area are complex and difficult. Although endoscopic approaches in skull base surgeries have progressed dramatically in recent years, there are few studies concerning these approaches.

Objectives: This study aimed to describe the endoscopic transcanal transcochlear approach (ETTA) to reach the internal auditory canal (IAC) in the cadaveric temporal bone specimen.

Methods: First, pre- and post-operative high resolution computed tomography (HRCT) images of the transcanal transcochlear pathway were obtained and 3D segmentation was performed using 3D Slicer software. The anatomic landmarks were identified using the endoscopic approach with and without navigation guide. Surgeries were done and the feasibility and challenges were evaluated.

Results: The internal auditory canal (IAC) was accessed via the ETTA. Anatomic landmarks were looked and a surgical corridor was identified with HRCT segmentations and images obtained during surgery.

Conclusions: We tried to increase our learning curve and extend surgical experience in lateral skull base surgery via minimally invasive ETTA. The increased endoscopic skills and the use of auxiliary tools such as a navigation guide may be helpful for successful access to the lateral skull base.
\end{abstract}

Keywords: Surgical Procedures, Endoscopy, Auditory Canal, Transcochlear, Anatomic Landmarks, Lateral Skull Base

\section{Background}

The lateral skull base, the anatomic boundary between the otolaryngology and the neurosurgery field, is always considered to be challenging for a surgeon (1). It contains several sensitive structures such as internal carotid artery, internal auditory canal (IAC), jugular bulb and the facial nerve. In this region, often it is required to perform an extensive surgical procedure (e.g. translabyrinthine, transcochlear, retro-sigmoid) to remove and treat benign lesions. These procedures are mainly performed based on the microscopic approach and sometimes associated with neurovascular manipulation and brain retraction, with complications such as seizure, facial nerve injury and intracranial complications (2).

The endoscopic approaches in skull base surgeries have progressed dramatically in recent years (3). It was first introduced in the 1990 s to investigate posterior epitympanum (4). Nowadays, it is mainly used for the management of middle ear lesions such as cholesteatoma $(5,6)$. Recently, lateral skull base surgeries were studied by Marchioni et al. (2). A learning curve and progression of training from lateral to medial have been described by Marchioni and Presutti group for the endoscopic technique as is demonstrated in Figure 1(7).

In the first phase, endoscopes were used to aid microscopic procedures, including tympanoplasties (5). With growing experience, the endoscopic techniques were used as the main tool in the surgical treatment of middle ear lesions such as cholesteatoma, using the microscope as a supplementary procedure $(5,6)$. After adequate experience in middle ear surgeries along with some preliminary studies on cadavers, some novel techniques were introduced for direct access to the inner ear via the external auditory canal (EAC), and to lateral skull base pathologies with endoscopic techniques (8). For the first time in 2015, the endoscopic transcanal approach was described as a 


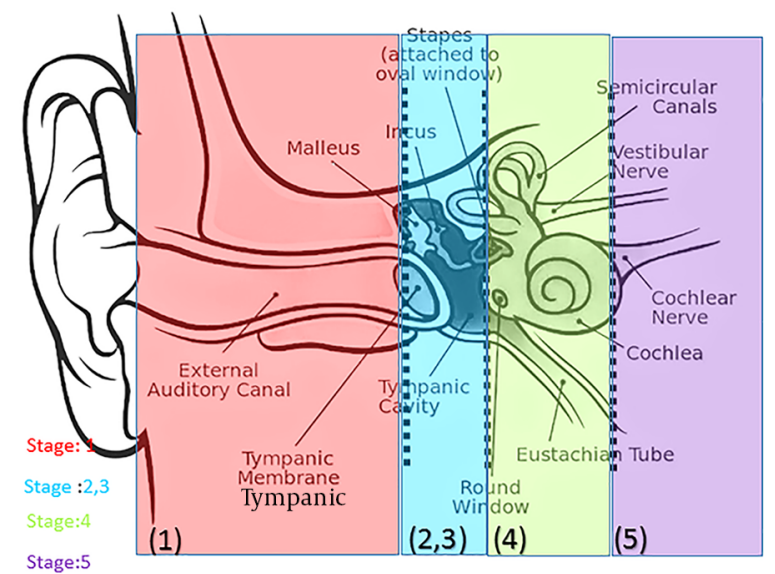

Figure 1. The learning curve of endoscopic techniques

surgical corridor to manage IAC lesions (2). Also, in a recent case series of 20 patients, the transcanal transpromontorial approach was used successfully for the removal of vestibular schwannoma (9).

The endoscopic approach has some advantages, including the potential for better visualization and outcome with less complication. Furthermore, it is accounted for less manipulation of brain or facial nerve (3). Despite these advantages, there are some limitations, including the necessity of adequate hand-eye coordination; the very limited space of the surgical field; visualization interrupt by bleeding, limited maneuvering due to some anatomical conditions (e.g. narrow EAC) (7).

Although the lateral skull base surgeries are well described and thoroughly studied for the classic microscopic techniques (step IV and V of Figure 1), there were few studies concerning the endoscopic approaches. It is due to the fact that the endoscopic procedures are new methods and they are dependent on the technology and instrument. Thus more studies are needed to describe endoscopic approaches of the lateral skull base and its landmarks and to increase the surgeons' skills.

\section{Objectives}

The aim of this study was a new modification for the endoscopic transcanal transcochlear approaches (ETTA) to reach the IAC in cadaveric specimens.

\section{Methods}

\subsection{Study Materials}

This study was conducted in Rasool-Akram Hospital in 2017-2018. The study was approved by the Ethics Committee with number IR.IUMS.FMD.REC.1397.149.

Three fresh cadaveric human temporal bones were acquired from the Forensic medical center and were anonymous. All specimens were placed in the anatomical position on the computed tomography (CT) scan bed and CT imaging was performed for each specimen preoperatively with spiral high-resolution CT (HRCT) for the surgical pathway 3D reconstruction and segmentation. Segmentation was done using 3D Slicer software. During these segmentations, vital structures located on the path of each endoscopic approach, such as the carotid artery, jugular vein, cochlea, and facial nerve were painted and then reconstructed. The surgical pathways, their anatomical location, and their adjacencies were determined.

Two of these cadaveric temporal bones were inserted in a head phantom and fixed with four screws (Figure 2). The endoscopic procedure was conducted with a Storz Professional Image Enhancement System (SPIES) endoscopy combined with an image-guided system (by the Parsis navigation system) and proper lenses $\left(0^{\circ}, 30^{\circ}, 2\right.$ millimeters and, 4 millimeters), bilaterally. Finally, the ETTA was performed on the temporal bones after gaining enough experience and skill with navigation guide. A repeated temporal bone CT scan was obtained postoperatively to confirm successful access to the lateral skull base.

\subsection{Surgical Technique}

In the endoscopic transcanal transcochlear approach (ETTA) (also named transcanal transpromontorial approach), EAC secretions were cleared using endoscopic view at first. An incision of the EAC was made at a position of 6 and 12 o'clock. The tympanic membrane was moved anterior or removed if necessary (Figure $3 \mathrm{~A}$ ). The EAC canaloplasty was performed if needed, using curette and drilling for better visualization and maneuvering of the inner wall of the middle ear (Figure 3B). Then malleus and incus were removed and the Stapes bone was carefully dislocated and separated from the oval window (Figure $3 \mathrm{C}$ ). This allowed a good view of the promontory region, round window, oval window, and the facial nerve (Figure 3D). In the ETTA, the medial othic capsule would be accessed via the oval window. The drilling was done in an anterior and posterior direction toward the round window. Then the round window and oval window were connected by drilling of the bone between them. The saccular fossa (spherical recess) was identified. After drilling 

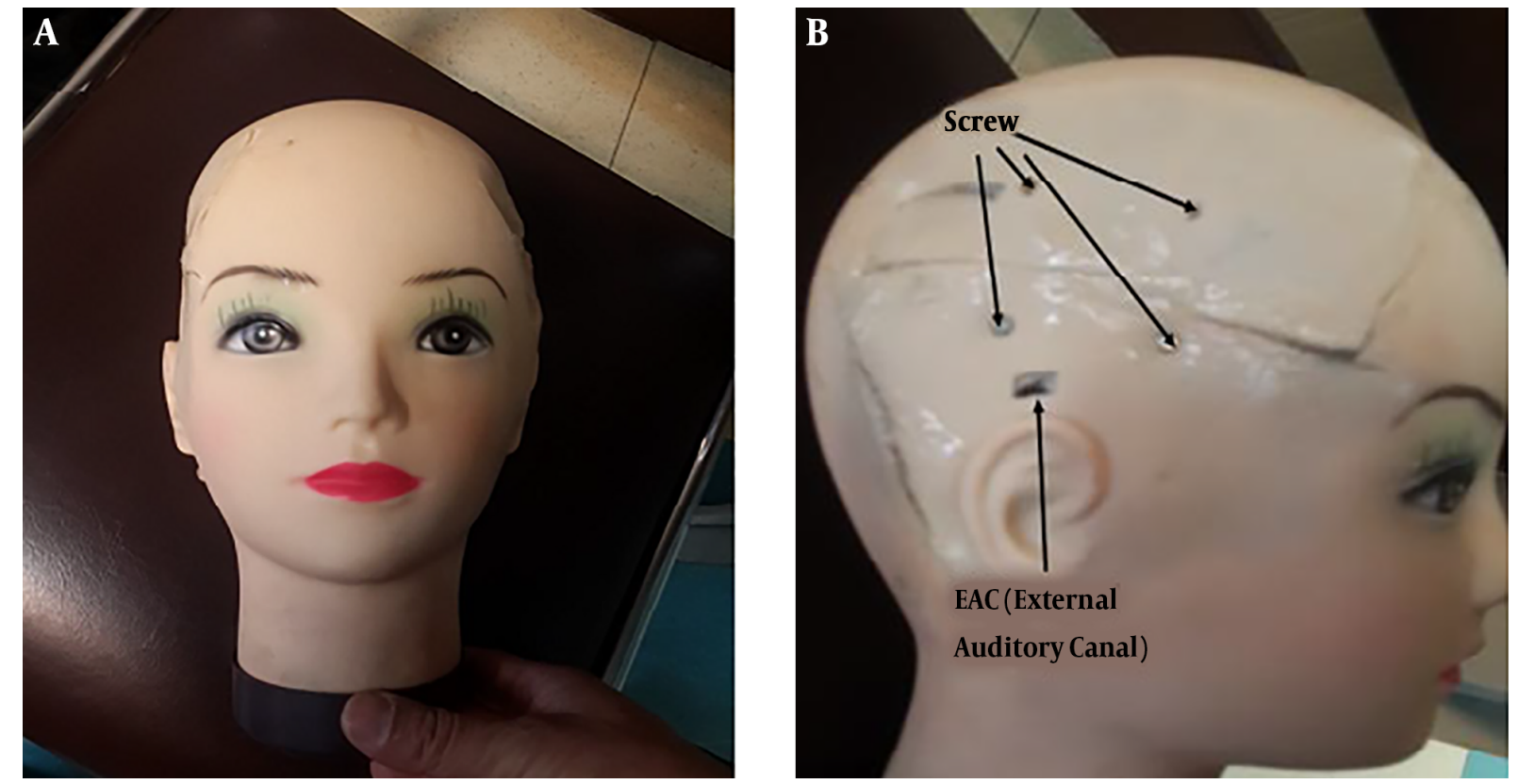

Figure 2. A, anterior and B, lateral views of phantom head

the oval window and the round window, the promontory was drilled to expose the cochlear turn and the facial nerve path and the IAC (Figure 3E). After identifying the basal turns of the cochlea, the drilling continued along its path to expose the middle and apical turns of the cochlea and modiolus (cochlear nerve pathway) (Figure 3F). The drilling was performed between the middle turn of the cochlea and the geniculate ganglion with concern regarding the intralabyrinthine segment of the facial nerve. The drilling and curettage continued along the medial and superior of the middle turn of the cochlea until the IAC fundus was identified (Figure 3G).

\section{Results}

The preoperative 3D segmentation of transcanal transcochlear pathway is demonstrated in Figure 4. As it is noted in the 3D segmentation, auditory ossicles are along its path. So, they should be removed to access IAC. Furthermore, transcanal transcochlear path was in the same direction as the EAC, and thus it needed limited canaloplasty.

In the ETTA, the medial othic capsule would be available via the oval window and saccular fossa (Spherical recess). Next step, the IAC fundus was identified between the middle turn of the cochlea and the geniculate ganglion. Also, the facial nerve pathway, anterior and superior to the
IAC is critical. The measured angle between IAC and the endoscopic transcanal transcochlear pathway was $27^{\circ}$ (Figure 4).

The ETTA was performed on the phantom head as was mentioned. The IAC fundus was accessed via this technique as it is demonstrated in Figure 5. The postoperative HRCT was obtained and the 3D segmentation was also performed using 3D Slicer software. According to this scan, the IAC was successfully accessed (Figure 6). It should be noted that with this approach, the auditory ossicles and labyrinthine were removed (total deafness) but the facial nerve was spared.

\section{Discussion}

Recently, endoscopic techniques have been introduced for treating lateral skull base lesions with several advantages over classic techniques, including the use of natural orifice (i.e. EAC), access to the IAC and minimal soft tissue dissection, and bone removal (10). Despite fascinating results, further studies are needed for better understanding of its landmark and increasing surgical knowledge and skills. In this study, we successfully reached the IAC with a minimally invasive transcanal endoscopic technique, i.e. ETTA, on the cadaveric temporal specimen.

The ETTA provides good visualization of the IAC, with high magnification of every structure within IAC and out- 

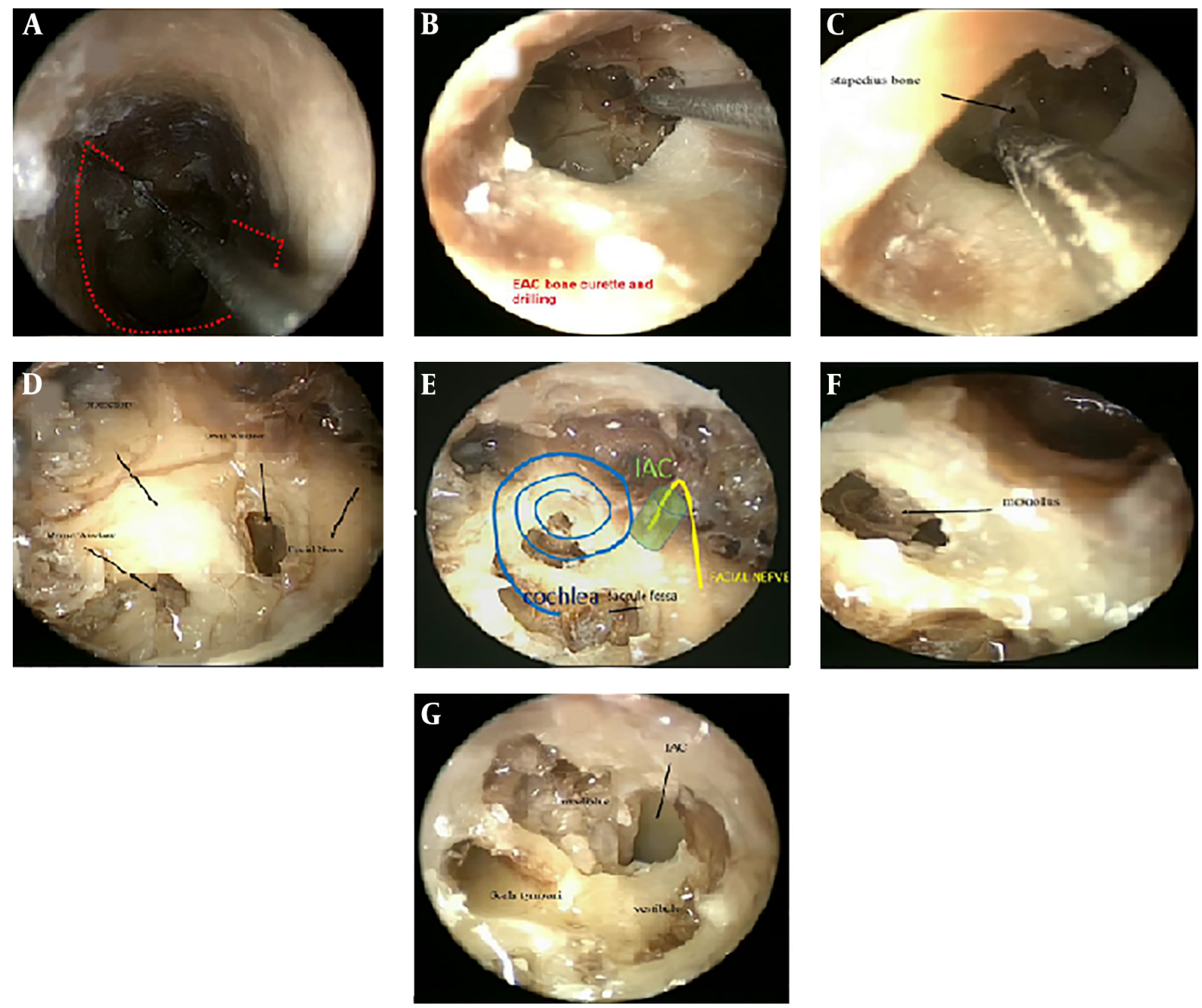

Figure 3. Transcochlear technique; A, EAC and tympanic membrane view; B, drilling and curettage of EAC; C, stapes bone removal; D, view of the promontory, round window, oval window, and the facial

side it, including the whole facial nerve path, and it requires limited bone drilling compared to traditional methods (11). Facial nerve re-routing, which is used in traditional techniques, is not necessary for ETTA. Thus less manipulation of this nerve could reduce the probability of its paralysis after surgery. Variable endoscopic angles could ensure no blind spot and therefore, fully resection of the underlying pathology (12). Using a navigation system, an endoscope holder and special endoscopic ear surgical set (with simultaneous suction and dissection instrument) could be helpful with ETTA efficacy. According to Salami et al. study (13), a piezoelectric device using low-frequency ultrasonic waves ( $25-30 \mathrm{kHz}$ ), which could be used for platinotomy, antro-atticotomy, mastoidectomy, and endo- auricular osteotomy, with variable headpiece and inserts could be helpful when performing ETTA and other endoscopic approaches. Hearing preservation is not achievable via traditional techniques also it is not possible with the $\operatorname{ETTA}(11,14)$.

Master et al. in their cadaveric study in 2016 (10) reported that IAC could be accessed via the endoscopic transcanal approach with a safe distance from vital neurovascular structures. During anatomical dissection of 19 temporal bones in this study, the mean distances from the carotid artery, jugular bulb, and middle fossa to the surgical path opening to the IAC were $4.1 \pm 1.5,6.4 \pm 2.5$, and $5.5 \pm 1.9 \mathrm{~mm}$, respectively. Marchioni et al. (11) conducted the first case series regarding exclusive ETTA access 


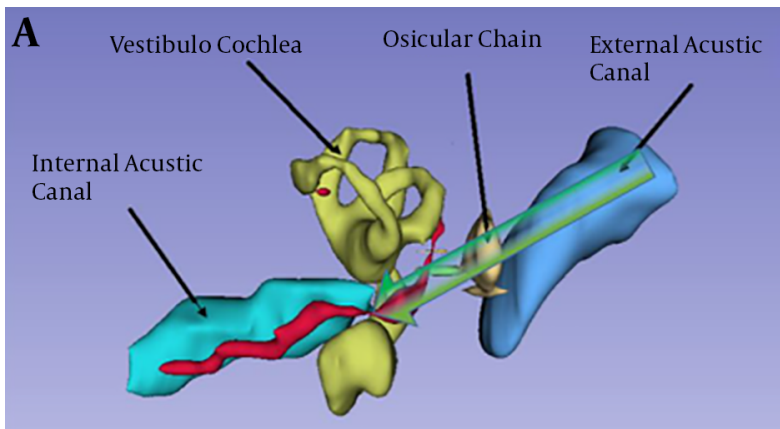

Pre op Segmentation of Transpromentory (Superior View)

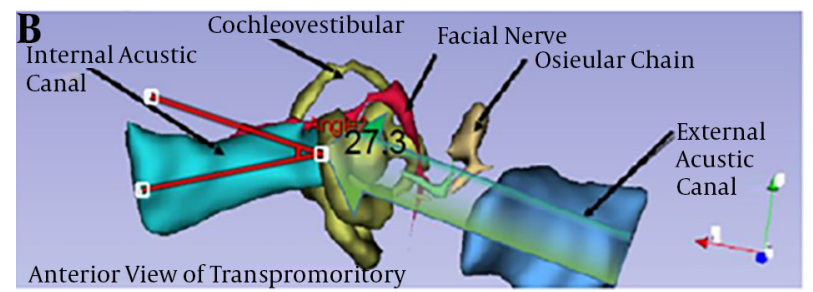

Figure 4. Preoperative 3D segmentation of temporal bone in the endoscopic transcanal transcochlear technique in A, superior and B, anterior view, demonstrating facial nerve pathway, IAC, EAC, auditory ossicles, and cochleovestibular.

to the IAC and its efficacy for vestibular schwannoma removal. In their study, 10 patients with vestibular schwannoma (Koos Grade I or II) underwent exclusive ETTA and gross total resection was achieved in all the patients, with an average operative time of $192 \mathrm{~min}$. Postoperatively, permanent facial nerve paresis/paralysis was observed in neither cases. According to another study by Marchioni et al. (15), although the hearing preservation is not possible with the ETTA, it is an effective approach for the resection of small intracanalicular acoustic neuroma with good postsurgical facial nerve function. Wick et al. (3) reported successful gross total resection of vestibular schwannomas in 4 patients ( 3 with Koos grade I and 1 with grade III) with a good long-term facial nerve function postoperatively. The mean hospital stay was 2.8 days in this study. Presutti et al. (16) concluded that expanded ETTA (using both endoscope and microscope, which allows bimanual dissection of the tumor and therefore, enhanced control of the surrounding neurovascular structures) was successful in the management of 10 patients with vestibular schwannoma Koos stage I or II with no facial dysfunction in the last follow-up. Marchioni et al. (9) described the expanded ETTA for the successful resection of vestibular schwannoma in patients with Koos grade II and III with no complication and good facial nerve result and concluded that this technique was a new approach that combined the advantages of a microscopic technique with the ones offered by the endoscope. Moon et al. (12) evaluated a modified ETTA with no EAC closure for the treatment of vestibular schwannomas and reported better cosmetic outcome compared to the original technique with total resection of the tumor in all cases.

The CSF leakage was observed less postoperatively with ETTA. Marchioni et al. (11) reported no CSF leakage after exclusive ETTA. In Presutti et al. study (16), 1 of 10 patients underwent expanded ETTA experienced postoperative CSF otorhinorrhea, which completely resolved in the follow-up period. Wick et al. (3) reported no postoperatively complication after ETTA on 4 patients with vestibular schwannomas. The mean percentage of this adverse effect was $10.3 \%$ for the retrosigmoid approach, 5.3\% for the middle cranial fossa approach, and 7.1\% for the translabyrinthine approach $(17,18)$. The lower risk of CSF leakage is because the ETTA is minimally invasive and with limited bone removal (9). The ETTA not only could minimize post-surgery complications but also could be a cost-benefit technique because it could shorten postoperative intensive care unit recovery and hospital stay $(9,11)$.

The important point of the present study is that for the lateral skull base surgeries through transcanal endoscopic techniques, increasing the surgeon's skill and knowledge and better identification of the anatomical landmarks are necessary. The navigation system and other auxiliary tools could be used to increase the surgical accuracy and for better evaluation of the vital structures and tumor extension. It should be noted that the lack of articles in cadaveric temporal could cause some study limitations. Considering the probability of less manipulation and complications with transcanal endoscopic techniques, further studies should be conducted to verify their feasibility in a patient with lateral skull base lesions.

\subsection{Conclusions}

We tried to increase our learning curve and extend surgical experience in lateral skull base surgery via a minimally invasive ETTA. Access to the lateral skull base and IAC is possible with classic microscopic methods; however, it is accompanied by several complications and morbidities. With the advancement in the technology of endoscopic devices, the endoscopic approaches may be used to treat the pathologies of IAC through the EAC. This requires increased endoscopic skills and the use of auxiliary tools such as navigation guide for successful access to the IAC and decreasing morbidities. 


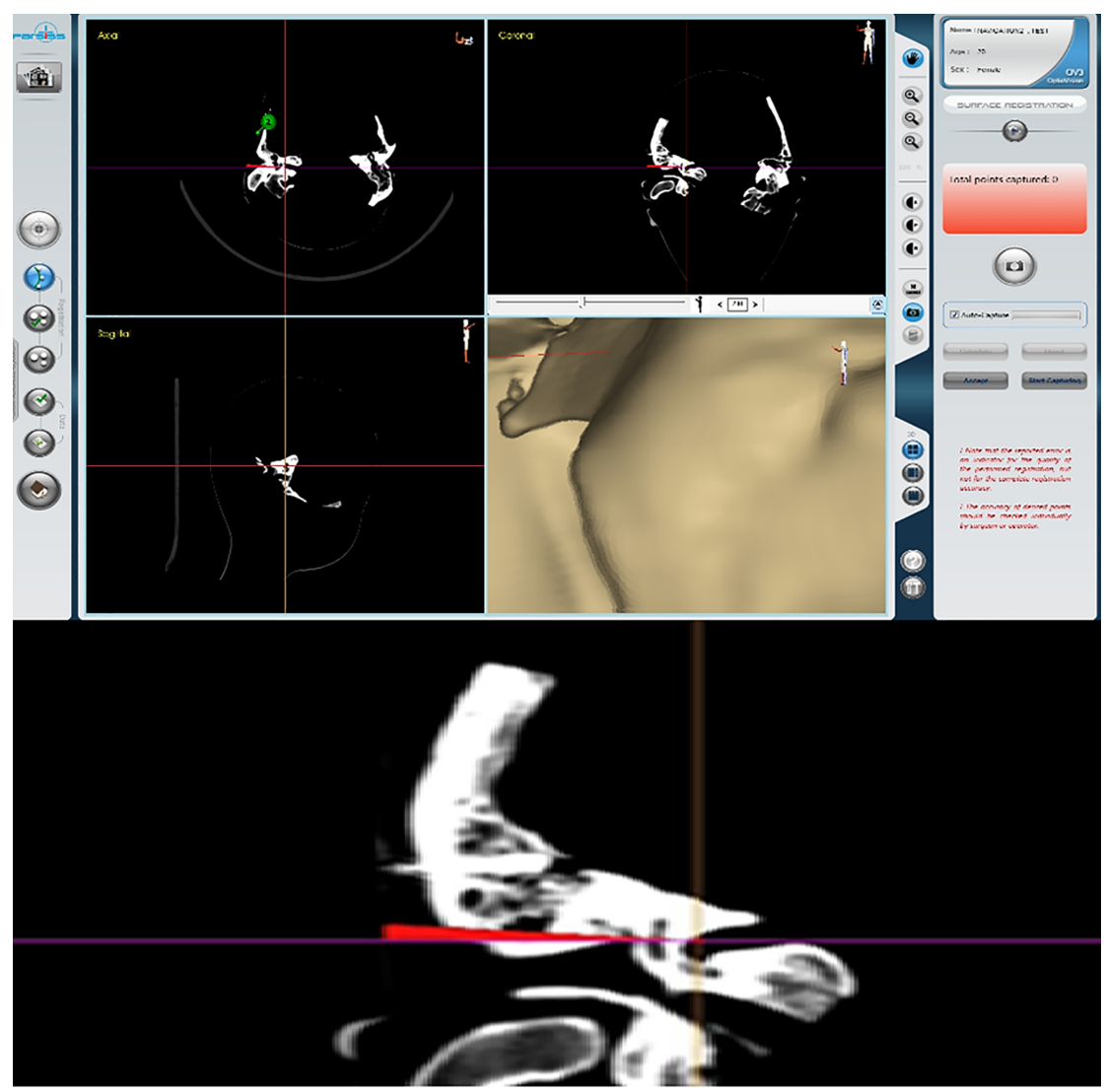

Figure 5. A, axial, sagittal, and coronal views of RT endoscopic transcanal transcochlear approach during surgery; B, closer view and access to the IAC
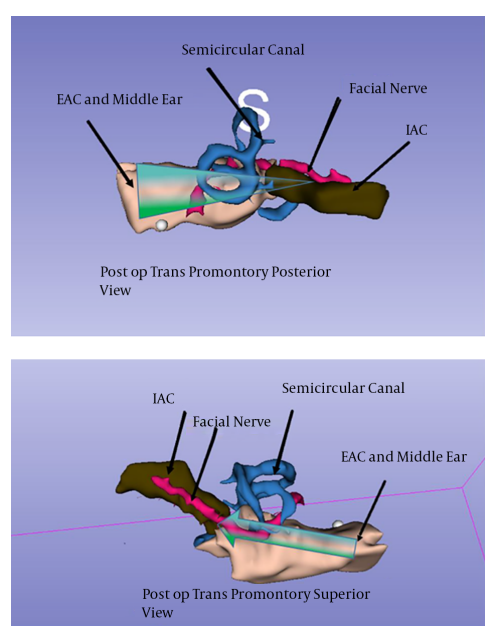

Figure 6. Postoperative 3D segmentation of the endoscopic transcanal transcochlear technique in $\mathrm{A}$, posterior and $\mathrm{B}$, superior view. EAC: external auditory canal; IAC: internal auditory cana

\section{Acknowledgments}

The present study was P.M's residency thesis for graduation in Otolaryngology-head and neck surgery specialty and supported by a grant from the Vice-Chancellor for Research, Iran University of Medical Sciences, Tehran, Iran.

\section{Footnotes}

Authors' Contribution: Saleh Mohebbi and Mohammad Papi developed the original idea and the protocol, abstracted, and analyzed data, wrote the manuscript, and other authors contributed to the development of the protocol, abstracted data, and prepared the manuscript.

Conflict of Interests: The authors reported no conflicts of interest.

Ethical Approval: The study was approved by the Ethics Committee with number IR.IUMS.FMD.REC.1397.149.

Funding/Support: This study was supported by Rasool Akram Hospital. 


\section{References}

1. Marchioni D, Bonali M, Presutti L. Transcanal endoscopic lateral skull base surgery. Oper Tech Otolayngol Head Neck Surg. 2017;28(1):57-64. doi: 10.1016/j.otot.2017.01.011.

2. Marchioni D, Alicandri-Ciufelli M, Rubini A, Presutti L. Endoscopic transcanal corridors to the lateral skull base: Initial experiences. Laryngoscope. 2015;125 Suppl 5:S1-13. doi: 10.1002/lary.25203. [PubMed: 25703066].

3. Wick CC, Arnaoutakis D, Barnett SL, Rivas A, Isaacson B. Endoscopic transcanal transpromontorial approach for vestibular schwannoma resection: A case series. Otol Neurotol. 2017;38(10):e490-4. doi: 10.1097/MAO.0000000000001588. [PubMed: 29135868].

4. Thomassin JM. The history and development of endoscopic ear surgery (EES). J Laryngol Otol. 2016;130(S3):S46-7. doi: 10.1017/s002221511600253x.

5. Presutti L, Marchioni D, Mattioli F, Villari D, Alicandri-Ciufelli M. Endoscopic management of acquired cholesteatoma: Our experience.J Otolaryngol Head Neck Surg. 2008;37(4):481-7. [PubMed: 19128580].

6. Tarabichi M, Nogueira JF, Marchioni D, Presutti L, Pothier DD, Ayache S. Transcanal endoscopic management of cholesteatoma. Otolaryngol Clin North Am. 2013;46(2):107-30. doi:10.1016/j.otc.2012.10.001. [PubMed: 23566900].

7. Alicandri-Ciufelli M, Marchioni D, Pavesi G, Canzano F, Feletti A, Presutti L. Acquisition of surgical skills for endoscopic ear and lateral skull base surgery: A staged training programme. Acta Otorhinolaryngol Ital. 2018;38(2):151-9. doi: 10.14639/0392-100X-1878. [PubMed: 29967560]. [PubMed Central: PMC6028814].

8. Marchioni D, Alicandri-Ciufelli M, Mattioli F, Nogeira JF, Tarabichi M, Villari D, et al. From external to internal auditory canal: Surgical anatomy by an exclusive endoscopic approach. Eur Arch Otorhinolaryngol. 2013;270(4):1267-75. doi: 10.1007/s00405-012-2137-x. [PubMed: 23010794].

9. Marchioni D, Carner M, Soloperto D, Bianconi L, Sacchetto A, Sacchetto L, et al. Expanded transcanal transpromontorial approach: A novel surgical technique for cerebellopontine angle vestibular schwannoma removal. Otolaryngol Head Neck Surg. 2018;158(4):710-5. doi: 10.1177/0194599818756592. [PubMed: 29405836].
10. Master A, Hamiter M, Cosetti M. Defining the limits of endoscopic access to internal auditory canal. J Int Adv Otol. 2016;12(3):298-302. doi: 10.5152/iao.2016.2998. [PubMed: 27897128].

11. Marchioni D, Alicandri-Ciufelli M, Rubini A, Masotto B, Pavesi G, Presutti L. Exclusive endoscopic transcanal transpromontorial approach: A new perspective for internal auditory canal vestibular schwannoma treatment. J Neurosurg. 2017;126(1):98-105. doi: 10.3171/2015.11.JNS15952. [PubMed: 26967786].

12. Moon IS, Cha D, Nam SI, Lee HJ, Choi JY. The feasibility of a modified exclusive endoscopic transcanal transpromontorial approach for vestibular schwannomas. J Neurol Surg B Skull Base. 2019;80(1):827. doi: 10.1055/s-0038-1667061. [PubMed: 30775216]. [PubMed Central: PMC6375732].

13. Salami A, Vercellotti T, Mora R, Dellepiane M. Piezoelectric bone surgery in otologic surgery. Otolaryngol Head Neck Surg. 2007;136(3):484-5. doi: 10.1016/j.otohns.2006.10.045. [PubMed: 17321883].

14. Kempfle J, Kozin ED, Remenschneider AK, Eckhard A, Edge A, Lee DJ. Endoscopic transcanal retrocochlear approach to the internal auditory canal with cochlear preservation: Pilot cadaveric study. Otolaryngol Head Neck Surg. 2016;154(5):920-3. doi: 10.1177/0194599816630979. [PubMed: 26932951]. [PubMed Central: PMC4960626].

15. Marchioni D, Carner M, Rubini A, Nogueira JF, Masotto B, Alicandri-Ciufelli $\mathrm{M}$, et al. The fully endoscopic acoustic neuroma surgery. Otolaryngol Clin North Am. 2016;49(5):1227-36. doi: 10.1016/j.otc.2016.05.014. [PubMed: 27565388].

16. Presutti L, Alicandri-Ciufelli M, Bonali M, Rubini A, Pavesi G, Feletti A, et al. Expanded transcanal transpromontorial approach to the internal auditory canal: Pilot clinical experience. Laryngoscope. 2017;127(11):2608-14. doi: 10.1002/lary.26559. [PubMed: 28271520].

17. Bennett M, Haynes DS. Surgical approaches and complications in the removal of vestibular schwannomas. Otolaryngol Clin North Am. 2007;40(3):589-609. ix-x. doi: 10.1016/j.otc.2007.03.007. [PubMed: 17544697].

18. Yamakami I, Ito S, Higuchi Y. Retrosigmoid removal of small acoustic neuroma: curative tumor removal with preservation of function. J Neurosurg. 2014;121(3):554-63. doi: 10.3171/2014.6.JNS132471. [PubMed: 24995779]. 